\title{
Avaliação da fitorremediação de solos contaminados com metais pelo capim braquiária e mostarda da Índia
}

\author{
Evaluation of phytoremediation of soils contaminated with metals by \\ surinam grass and India mustardcrassipes (hyacinth)
}

\author{
Maristela Silva Martinez | Daniela Ferreira Cardoso Cruvinel | Danilo Moraes Baratto
}

Data de entrada: 20/09/2011 | Data de aprovação: 03/09/2012

\section{Resumo}

Neste trabalho de pesquisa foram avaliadas a capacidade do capim braquiária (Braquiária Decumbens) e mostarda da índia (Brassica Juncea) de extrairem metais de solo L 25, Latossolo Roxo. O delineamento experimental foi realizado em blocos com esquema fatorial 2 espécies x 4 doses de metais x 4 repetições. As doses de metais foram baseadas nos valores de Intervenção para solos agrícolas da decisão no 195-2005-E, de 23/11/2005 da CETESB, aplicadas na forma de sais com as seguintes concentrações em mg kg-1: dose zero; dose I - adição combinada de 450 de Zn, 3 de Cd, 180 de Pb, 70 de Ni e 150 de Cr; dose II - 2 vezes a dose I e dose III- 3 vezes a dose I. A colheita foi realizada após 180 dias do plantio. A mostarda da índia adaptou-se a todas as concentrações aplicadas. O zinco foi o metal que apresentou maior remoção do solo contaminado pelas duas espécies. Para contaminação dose I a mostarda da índia se mostrou melhor na remoção de cromo (37\%), chumbo (39\%) e níquel (68\%), enquanto o capim braquiária removeu melhor os metais chumbo (40\%), cromo (62\%) e níquel (88\%). Ambas espécies revelaram ser boas opções para fitorremediação de solos contaminados com metais pesados.

Palavras-chave: Fitoextração, Brassica Juncea, Brachiaria Decumbens, Poluição Do Solo.

\begin{abstract}
We evaluated the ability of pasture grass and Indian mustard plants grown in eight-liter pots to extract metals from the soil latosol. The experimental design consisted in randomized blocks with factorial 2 species $x 4$ metal doses $x 4$ replications. The metal doses were based on values recommended by the CETESB interventional decision number 195-2005-E issued on 23rd November 2005 and were applied in the form of inorganic salts with the following concentrations in $\mathrm{mg} \mathrm{kg}$-1: dose zero - without addition of any metal; dose I - combined addition of $450 \mathrm{Zn}, 3 \mathrm{Cd}, 180 \mathrm{~Pb}, 70 \mathrm{Ni}$, and $150 \mathrm{Cr}$; dose IIcombined addition of $900 \mathrm{Zn}, 6 \mathrm{Cd}, 360 \mathrm{~Pb}, 140 \mathrm{Ni}$, and $300 \mathrm{Cr}$; dose III- combined addition of 1350 $\mathrm{Zn}, 9 \mathrm{Cd}, 540 \mathrm{~Pb}, 210 \mathrm{Ni}$, and $450 \mathrm{Cr}$. The crop was harvested 180 days after planting. Brassica juncea was the species that best adapted to all the applied concentrations. Zinc was the element that showed greatest soil removal in soil contaminated by both species. For the contamination dose I Brassica juncea was the best for chromium (37\%), lead (39\%), and nickel (68\%) removal, while Brachiaria decumbens removed the metals lead (40\%), chromium (62\%), and nickel (88\%) the most effectively. Both species were excellent choices for the phytoremediation of soils contaminated with heavy metals.
\end{abstract}

Key-words: Phytoextraction, Brassica Juncea, Brachiaria Decumbens, Soil Pollution.

Maristela Silva Martinez*

Bacharel em Química, Doutora em Físico-Química pelo IQ da USP São Carlos. Professora Titular da Universidade de Ribeirão Preto, UNAERP. Orientadora do Programa de Mestrado em Tecnologia Ambiental da UNAERP.

\section{Daniela Ferreira Cardoso Cruvinel} Bióloga, Mestre em Tecnologia Ambiental pela Universidade de Ribeirão Preto. Docente do Instituto Federal de Educação, Ciência e Tecnologia do Sul de Minas Gerais - Campus Muzambinho.

\section{Danilo Moraes Baratto}

Engenheiro Químico pela Universidade de Ribeirão Preto. Responsável pelo Laboratório de Química Agrícola da Universidade de Ribeirão Preto.

*Endereço para Correspondência: Avenida Costábile Romano, 2201 Bloco D, Sala 07 - Ribeirão Preto, SP - CEP 14098-380 - Telefone 55(16)36036784 - e-mail: mmartinez@unaerp.br 


\section{Introdução}

A contaminação de solos e água com poluentes químicos representa grande problema ambiental com implicações para a saúde humana e animal. A crescente industrialização e urbanização têm exposto animais e vegetais a elementos químicos potencialmente tóxicos. Entre estes, os metais pesados representam o maior contaminante industrial dos ecossistemas (Gaetke e Chow, 2003). O estado de São Paulo possui 1396 áreas contaminadas segundo Relatório da qualidade do solo do estado de São Paulo de Novembro de 2009 (http://www.cetesb.sp.gov.br/solo/publicações-e-Relatórios/1-Publicações-/-Relatórios)

$\mathrm{O}$ tratamento de áreas degradadas é exigência da lei (Lei $\mathrm{N}^{\circ}$ 6.938, de 31 de agosto de 1981, regulamentada pelo Decreto $\mathrm{N}^{\circ}$ 99.274/90) e, além disso, assumiu o status de compromisso social que precisa ser realizado. Existem inúmeras tecnologias para remediação de solos de áreas contaminadas, as quais envolvem processos químicos, processos físicos e processos biológicos, sendo que todos requerem tecnologias muitas vezes complicadas e de custo elevado. As técnicas de remediação têm por base processos de engenharia direcionados a aumentar a capacidade de extração dos contaminantes com a aplicação de calor, surfactantes, ácidos e manipulação física do solo contaminado. Os processos de remediação podem ser divididos em dois grupos de acordo com o local de operação, in situ ou ex situ (Huang e Cunnigham, 1996).

Os métodos in situ são os mais apropriados, entretanto sua eficiência depende de algumas variáveis como características do local, concentração dos metais pesados a serem removidos, além do uso final do ambiente contaminado (Cunningham et al., 1996; Siqueira et al., 1999). Esses métodos têm como objetivo imobilizar os metais pesados, para removê-los. O tratamento de áreas contaminadas por metais pesados através de processos físico-químicos, além de apresentarem custos elevados, freqüentemente causa danos colaterais indesejados, como a necessidade de remoção de parte do solo. (Mulligan et al., 2001).

Por esse motivo, o uso da fitorremediação, tecnologia baseada no uso

de plantas capazes de absorver e acumular em seus tecidos metais pesados e outros compostos químicos do solo e da água, ganhou grande interesse durante as últimas década (Maine et al., 2004; Silva et al., 2006; Skinner et al., 2007;
Agunbiade et al., 2009).

As substâncias alvo da fitorremediação incluem metais ( $\mathrm{Pb}, \mathrm{Zn}, \mathrm{Cu}, \mathrm{Ni}, \mathrm{Hg}$, $\mathrm{Se}, \mathrm{Cr}, \mathrm{Cd}$ ), compostos inorgânicos, elementos químicos radioativos (U, Cs, Sr), hidrocarbonetos derivados de petróleo, praguicidas e herbicidas, explosivos (TNT, DNT), solventes clorados (TCE, PCE) e resíduos orgânicos industriais (PCP, HPA), entre outros (Cunningham et al, 1996; Wei et al, 2008; Shengwei et al, 2009; ).

As plantas hiperacumuladoras são altamente especializadas em acumular ou tolerar concentrações elevadas de metais como: $10.000 \mathrm{mg}$ kg-1 de Zn e Mn; 1.000 mg kg-1 de Pb, Ni e $\mathrm{Cu} ; 100$ mg kg-1 de Cd (Raskin et al., 1994). Até o momento foram identificadas cerca de 400 plantas hiperacumuladoras, sendo a maioria originária de áreas contaminadas da Europa, Estados Unidos, Nova Zelândia e Austrália (Khan et al., 2000). Essas plantas hiperacumuladoras pertencem às famílias Brassicaceae, Fabaceae, Euphorbiaceae, Asteraceae, Lamiaceae e Scrophulariaceae (Garbisu e Alkorta, 2001). Algumas plantas possuem potencial para extrair vários metais do solo, outras são mais específicas. A Brassica juncea possui potencial para remediar solos com altos teores de $\mathrm{Pb}, \mathrm{Cr}, \mathrm{Cd}, \mathrm{Cu}, \mathrm{Ni}, \mathrm{Zn}, \mathrm{Sr}, \mathrm{B}$ e Se; Thlaspi caerulescens para fitorremediar $\mathrm{Cd}$, $\mathrm{Ni}$ e Zn; Helianthus annuus, Nicotiana tabacum e Alyssum wufenianum para extrair Ni (USEPA, 2000). A fitorremediação de solos contaminados com metais parte de dois principais processos: fitoestabilização, que consiste na imobilização de metais no solo ou nas raízes, reduzindo assim a sua mobilidade e biodisponibilidade e fitoextração, que identifica a captação de contaminantes do solo e sua translocação das raízes para a parte aérea da planta (Pivetz, 2001).

A fim de alcançar uma boa eficiência no processo de fitorremediação as plantas devem acumular grandes quantidades de metais pesados, tolerar solos com poluição, e também produzir uma grande quantidade de biomassa nas condições de contaminação (McGrath et al., 2002).

Dessa forma, a investigação do desenvolvimento das plantas em diferentes níveis de contaminação por metais se torna relevante.este trabalho foi avaliada a fitorremediação de solos artificialmente contaminado com os metais $\mathrm{Zn}$, $\mathrm{Cd}, \mathrm{Pb}, \mathrm{Cr}$ e Ni pelos vegetais capim braquiária (Brachiaria decumbens) e mostarda da índia (Brassica juncea). Foram aplicadas diferentes do- 
ses de metais a fim de verificar a adaptação de cada planta a diferentes graus de contaminação do solo.

\section{Materiais e Métodos}

Os ensaios foram realizados em recipientes de 10 L contendo em média $8 \mathrm{~kg}$ de solo típico da região de Ribeirão Preto-SP (Latossolo Roxo). O solo foi preparado com terra de barranco (terra virgem), oriunda de Guaxupé - MG, substrato orgânico, adubo do tipo supersimples e calcário. Os experimentos foram delineados em blocos ao acaso com esquema fatorial 2 X 4, com 4 repetições..

Os vegetais estudados foram capim braquiária e Mostarda da Índia. Estes vegetais foram selecionados por apresentarem características fitorremediadoras (Hong-Ming et al, 2009). As sementes de mostarda da Índia utilizadas foram fornecida pela Isla Sementes e as de capim braquiária foram fornecidas pela AgroLima Sementes.

Para contaminação do solo foram utilizados sais com grau analítico de cada um dos metais distribuídos em diferentes concentrações, de forma a fornecer múltiplos dos Valores de Intervenção preconizados pela CETESB (2005). A Tabela 1 apresenta os valores de intervenção para cada metal estudado, os sais aplicados e as 3 concentrações de contaminação efetuadas dados em miligramas de metal por quilograma de solo seco.

Os vasos foram mantidos em área coberta com iluminação natural e rega diária. A temperatura média durante o período de experimento foi de $25 \pm 3$ oC.A coleta dos vegetais foi realizada após 180 dias do plantio. Os vegetais foram coletados, lavados, armazenados em sacos de papel e mantidos em estufa a 600C até secagem total. Em seguida cada amostra foi triturada em moinho e passada por uma placa perfurada com furos de $1 \mathrm{~mm}$ de diâmetro e $17 \%$ de área aberta. A massa seca foi então pesada e digerida para análise dos metais. Após a coleta dos vegetais, o solo de cada vaso foi quarteado, coletado e digerido para análise dos metais. Tanto para a digestão do metal como para a digestão do solo foi utilizada a digestão nitro-perclórica segundo Malavolta (1997).

A análise dos metais foi realizada em triplicata utilizando a técnica de Espectrofotometria de absorção atômica (EAA) em um espectrofotômetro Perkin Elmer (AAnalyst100) com chama de ar/ acetileno e lâmpada de catodo oco. Foram utilizadas soluções padrões com reagentes de grau analítico de cada um dos metais para construção de cada curva de calibração. Os limites de quantificação para todos os metais foi de 0,001 mg.L-1. Com os resultados obtidos foi criado um parâmetro para avaliar o potencial acumulador de cada metal por cada uma das plantas. O parâmetro foi calculado pela divisão da massa de metal acumulado pela massa de matéria seca obtida de cada planta e pelo tempo de plantio em dias e multiplicado por 30 para resultar em g de metal/grama de massa seca/mês.

\begin{tabular}{|c|c|c|c|c|c|}
\hline \multirow{2}{*}{ Metal } & \multirow{2}{*}{ Sal aplicado } & \multirow{2}{*}{$\begin{array}{l}\text { Valor de Inter- } \\
\text { venção } \mathrm{mg} \cdot \mathbf{k g}^{-1}\end{array}$} & \multicolumn{3}{|c|}{ Contaminação mg.kg ${ }^{-1}$} \\
\hline & & & I & II & III \\
\hline Chumbo & $\mathrm{Pb}(\mathrm{NO} 3)^{2}$ & 180 & 180 & 360 & 540 \\
\hline Cadmio & $\mathrm{CdCl}^{2}$ & 3 & 3 & 6 & 9 \\
\hline Cromo & $\mathrm{K}_{2} \mathrm{Cr}_{2} \mathrm{O}_{7}$ & 150 & 150 & 300 & 450 \\
\hline Níquel & $\mathrm{Ni}\left(\mathrm{NO}_{3}\right)_{2}$ & 70 & 70 & 140 & 210 \\
\hline Zinco & $\mathrm{ZnCl}_{2}$ & 450 & 450 & 900 & 1350 \\
\hline
\end{tabular}

Tabela 1 - Dados dos valores de intervenção e da contaminação realizada em cada um dos tratamentos em mg do metal por $\mathrm{kg}$ de solo seco. 


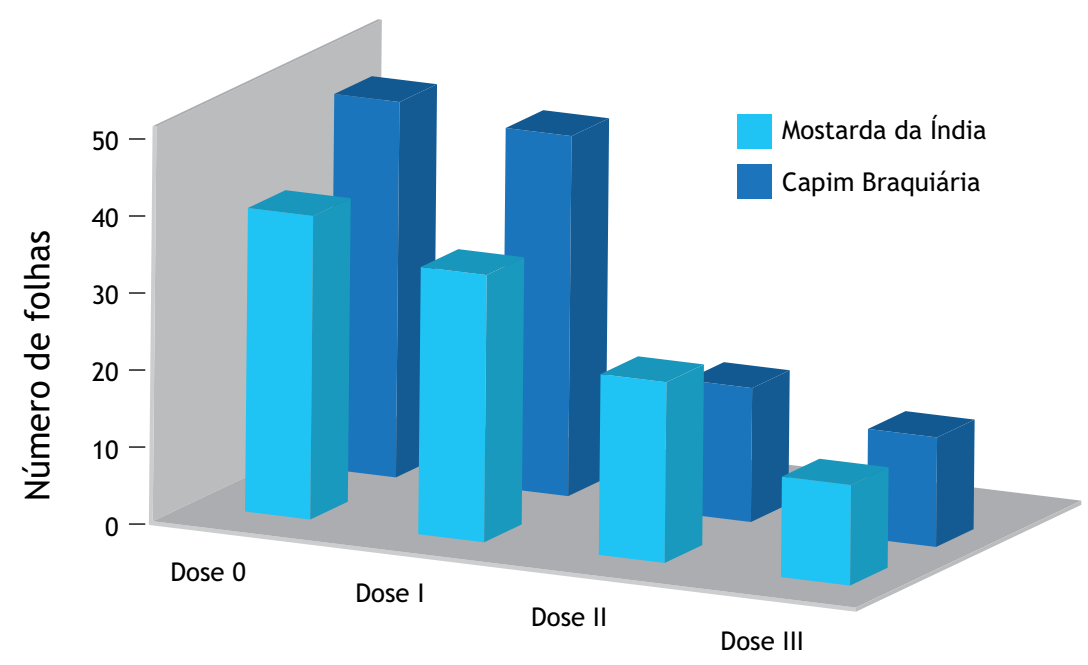

Figura 1: Número médio de folhas presentes nos vasos de Brachiaria decumbens após 175 dias do plantio e nos vasos de mostarda da Índia após 104 dias do plantio

\section{Resultados e Discussões}

\section{Desenvolvimento das plantas - Sintomas visu-} ais

O capim braquiárias se desenvolveu em 12 dos 16 recipientes, sendo 4 sem contaminação, 4 contaminados com dose I , 3 com dose II e apenas 1 com dose III. Observaram-se diferenças significativas no desenvolvimento das plantas, que apresentaram redução no número de folhas nos vasos com contaminação II e III. A mostarda da Índia se desenvolveu na maioria dos recipientes, exceto em dois vasos com dose III, mostrando-se melhor adaptada às condições estudadas . A Fi- gura 1 apresenta o gráfico com o número médio de folhas de cada espécie em cada dose de metal aplicada.

Observa-se que a dose I tanto para o capim braquiária quanto para a mostarda da Índia não provocou redução significativa no número de folhas. Já a dose II levou a uma redução de $67 \%$ no número de folhas da Braquiária e 43\% da Brassica. Nos vasos com o dose III a redução foi de $73 \%$ na Braquiária e 68\% para a Brassica. Mesmo apresentando bom desenvolvimento, as plantas apresentaram algumas deformações como manchas esbranquiçadas nas folhas, principalmente

\begin{tabular}{c|c|c|c|c}
\multirow{2}{*}{ Solo de cultivo } & \multicolumn{2}{|c|}{ Capim braquiária } & \multicolumn{2}{c}{ Mostarda da Índia } \\
\cline { 2 - 5 } & $\begin{array}{c}\text { Massa de vegetal } \\
\text { seco (g) }\end{array}$ & Desvio médio & $\begin{array}{c}\text { Massa de vegetal } \\
\text { seco (g) }\end{array}$ & Desvio médio \\
\hline Não contaminado & 22,3 & 2,7 & 11,0 & 1,8 \\
\hline grau I & 19,5 & 2,1 & 11,1 & 1,2 \\
\hline grau II & 12,1 & 2,3 & 8,4 & 0,6 \\
\hline grau III & 4,2 & - & 7,0 & 0,7 \\
\hline
\end{tabular}

Tabela 2 - Média dos pesos das massas vegetais obtidas em cada grau de contaminação 


\begin{tabular}{c|c|c|c|c|c}
\multirow{2}{*}{ Metal } & \multicolumn{2}{|c|}{ Contaminação 1} & \multicolumn{2}{c|}{ Contaminação 1 } & Contaminação 3 \\
\cline { 2 - 5 } & $\mathrm{mg} / \mathrm{kg}$ & $\mathrm{mg} / \mathrm{kg}$ & $\mathrm{mg} / \mathrm{kg}$ & $\mathrm{mg} / \mathrm{kg}$ & $\mathrm{mg} / \mathrm{kg}$ \\
\cline { 2 - 6 } & $\begin{array}{c}\text { Capim } \\
\text { Braquiária }\end{array}$ & $\begin{array}{c}\text { Mostarda da } \\
\text { Índia }\end{array}$ & $\begin{array}{c}\text { Capim } \\
\text { Braquiária }\end{array}$ & $\begin{array}{c}\text { Mostarda da } \\
\text { Índia }\end{array}$ & $\begin{array}{c}\text { Mostarda da } \\
\text { Índia }\end{array}$ \\
\hline $\mathrm{Cd}$ & $0,70 \pm 0,05$ & $0,90 \pm 0,05$ & $5,0 \pm 0,9$ & $1,5 \pm 0,3$ & $7,0 \pm 0,9$ \\
\hline $\mathrm{Zn}$ & $120 \pm 4$ & $191 \pm 4$ & $178 \pm 2$ & $293 \pm 5$ & $320 \pm 9$ \\
\hline $\mathrm{Ni}$ & $23 \pm 2$ & $10,5 \pm 0,5$ & $35 \pm 2$ & $16 \pm 1$ & $24 \pm 1$ \\
\hline $\mathrm{Cr}$ & $56 \pm 6$ & $27 \pm 4$ & $13,0 \pm 0,5$ & $75 \pm 8$ & $0,10 \pm 0,05$ \\
\hline
\end{tabular}

Tabela 3 - Resultados das concentrações dos metais em mg de metal por kg de vegetal obtidos nos vegetais capim braquiária e mostarda da Índia para a contaminação I, II e III

nos vasos com maior concentração dos metais. Este fato aponta uma elevada fitotoxidade das plantas que se desenvolvem em solos com altas concentrações de metais pesados. Este tipo de comportamento foi observado por Zeitouni et al. (2007) no estudo da capacidade das plantas de mamona, girassol, pimenta da amazônia e tabaco em extrair Zn e Cd de um Latossolo Vermelho-Amarelo distrófico, onde foi observado uma redução das folhas e aparecimento de manchas nas mesmas em função da fitotoxidade.

\section{Produção de Matéria Seca}

A Tabela 2 apresenta o peso total do vegetal seco obtido em cada uma das contaminações aplicadas e o desvio médio.

Com os dados apresentados na Tabela 2, observa-se que, conforme a contaminação aumentou, houve uma redução significativa na massa seca do capim braquiária. Com dose I houve redução de $12,8 \%$, já na dose II, $45,8 \%$ e na dose III, a redução foi de $81,2 \%$. Isso demonstra que a contaminação por metais afeta o desenvolvimento da planta, prejudicando o processo de fitorremediação nessa espécie. Esta redução de massa seca deve ser decorrente da fitotoxidade dos metais no capim braquiária.

A mostarda da Índia não apresentou redução de massa para contaminação dose I. Já para a dose II, a redução foi de $23,6 \%$ e para dose III, de $36,4 \%$. Este fato vem corroborar a boa perspec- tiva de aplicação deste vegetal na fitorremediação de solos contaminados por metais (USEPA, 2000).

\section{Metais presentes na matéria seca}

A Tabela 3 apresenta a concentração dos metais na matéria seca dos vegetais em cada dose de contaminação.

Comparando os resultados das concentrações dos metais em cada um dos vegetais, em cada tratamento, observa-se que a mostarda da Índia apresenta melhor acúmulo de $\mathrm{Zn}$ e $\mathrm{Cd}$, enquanto o capim braquiária acumulou maior quantidade de Ni, $\mathrm{Cr}$ e Pb. Na dose de contaminação II a mostarda da Índia continuou melhor para acumular Zn e superou o capim braquiária no acúmulo de $\mathrm{Cr}$ e $\mathrm{Pb}$.

\section{Metais presentes no solo}

A Tabela 4 apresenta as médias da concentração de cada metal (contaminação dose I, dose II e dose III) no solo onde estavam cultivados os vegetais mostarda da Índia e capim braquiária.

Para o grau de contaminação I, houve redução de cerca $40 \%$ do Zn presente no solo de ambos os cultivos. A braquiária se mostrou mais eficiente na redução das concentrações de Ni e Cr (88\% e $62 \%$ respectivamente). Já a mostarda da índia foi mais eficiente na remoção do $\mathrm{Cd}$, com remoção de $17,3 \%$, sendo que o capim braquiária removeu apenas cerca de $7 \%$. 
mg de metal por kg de solo

\begin{tabular}{|c|c|c|c|c|c|}
\hline \multicolumn{6}{|c|}{ Dose I } \\
\hline Espécie Cultivada & $\mathrm{Cd}$ & $\mathrm{Ni}$ & $\mathrm{Cr}$ & $\mathrm{Zn}$ & $\mathrm{Pb}$ \\
\hline Inicial & 3,00 & 70 & 150 & 450 & 180 \\
\hline Capim Braquiária & $2,78 \pm 0,07$ & $8,2 \pm 0,7$ & $57 \pm 14$ & $280 \pm 21$ & $107 \pm 18$ \\
\hline Mostarda da Índia & $2,48 \pm 0,08$ & $22 \pm 2$ & $94 \pm 12$ & $276 \pm 26$ & $127 \pm 25$ \\
\hline \multicolumn{6}{|c|}{ Dose II } \\
\hline Inicial & 6,00 & 140 & 300 & 900 & 360 \\
\hline Capim Braquiária & $2,4 \pm 0,7$ & $23 \pm 2$ & $242 \pm 30$ & $724 \pm 99$ & $299 \pm 38$ \\
\hline Mostarda da Índia & $3,6 \pm 0,3$ & $45 \pm 5$ & $203 \pm 26$ & $575 \pm 56$ & $224 \pm 23$ \\
\hline \multicolumn{6}{|c|}{ Dose III } \\
\hline Inicial & 9,00 & 210 & 450 & 1350 & 540 \\
\hline Mostarda da Índia & $6,5 \pm 2$ & $86 \pm 21$ & Parcela perdida & $1091 \pm 213$ & $386 \pm 87$ \\
\hline
\end{tabular}

Tabela 4 - Resultados das concentrações dos metais no solo onde foram cultivados os vegetais mostarda da Índia e capim braquiária

Remoção $\left(\mathrm{g} \cdot \mathrm{kg}^{-1} \cdot \mathrm{mês}^{-1}\right)$

\begin{tabular}{l|c|c|c|c|c|c}
\hline \multicolumn{7}{c}{ Dose I } \\
\hline Espécie Cultivada & Cd & Ni & Cr & Zn & Pb \\
\hline Capim Braquiária & 0,004 & 1,058 & 1,592 & 2,910 & 1,250 \\
\hline Mostarda Da Índia & 0,016 & 1,438 & 1,677 & 5,211 & 1,578 \\
\hline Brachiaria Decumbes & 0,099 & 3,223 & 1,598 & 4,848 & 1,680 \\
\hline Mostarda Da Índia & 0,114 & 4,505 & 4,599 & 15,41 & 6,449 \\
\hline
\end{tabular}

Tabela 5 - Parâmetros de remoção de cada metal pelo capim braquiária e pela Mostarda da Índia dados em gramas de metal por $\mathrm{kg}$ de vegetal por um período médio de 30 dias 
Verificou-se que, após o aumento da contaminação para a dose II, o solo onde estava cultivada a mostarda da índia obteve uma redução maior na quantidade dos metais $\mathrm{Cr}$, $\mathrm{Zn}$ e $\mathrm{Pb}$. Já o solo onde estava cultivado o capim braquiária, obteve uma redução maior na quantidade dos metais $\mathrm{Cd}$ e $\mathrm{Ni}$, confirmando os dados obtidos nas análises dos vegetais.

A fim de criar um parâmetro para definir o potencial acumulador de cada vegetal, foi calculada a massa de metal acumulada por kg de vegetal em um período médio de 30 dias (Tabela 5).

Nenhuma das espécies estudadas se mostarram boas acumuladoras para o cádmio e as duas espécies apresentaram resultados satisfatórios para remoção de zinco, cromo, níquel e chumbo. A mostarda da Índia apresentou no geral melhores resultados quando comparada com o outro vegetal estudado, obtendo-se uma remoção mais elevada de Zn (5,21 g/kg.mês) enquanto o capim braquiária obteve 2,91 g. kg-1.mês-1. Para a dose II, observou-se que no solo onde estava cultivada a mostarda da Índia houve uma maior remoção dos metais $\mathrm{Zn}, \mathrm{Pb}, \mathrm{Cr}$ e Ni. Já no solo onde estava cultivada o capim braquiária, houve uma maior remoção dos metais zinco, níquel e chumbo. Comparando os resultados obtidos pelos dois vegetais, conclui-se que a mostarda da Índia apresentou uma remoção de 15,41 g. kg-1 mês-1 para o zinco enquanto o capim braquiária obteve 4,84 g. kg-1.mês-1 para este metal. Os resultados continuaram insatisfatórios para a remoção de cádmio e a mostarda da Índia apresentou melhores resultados que o capim braquiária.

Na dose III somente a mostarda da Índia se desenvolveu. Comparando o comportamento dos dois vegetais nos solos contaminados com diferentes concentrações (I, II e III), pode-se observar o melhor desempenho da mostarda da Índia na maioria dos metais analisados. Para o metal cádmio não houve avanço na remoção pelos dois vegetais.

É importante destacar o comportamento de remoção de metais pela mostarda da Índia que se manteve inalterado mesmo aumentando a concentração dos metais de dose II para dose III. Este fato indica uma boa resistência deste vegetal em solos com elevadas concentrações de metais, mostrando a viabilidade de sua aplicação na fitorremediação de solos deste tipo.

Esses resultados se mantiveram para o cádmio, chumbo e níquel. Houve uma diminuição de remoção para o zinco (de 15,41 g kg-1 mês-1 no grau II de contaminação para 10, 27 g kg-1 mês-1 no grau III de contaminação).

\section{Conclusões}

A mostarda da Índia foi a espécie que melhor se desenvolveu em solos contaminados com metais, se mostrando um vegetal com excelente potencial fitorremediador para $\mathrm{Zn}, \mathrm{Cr}, \mathrm{Pb}$ e $\mathrm{Ni}$. O capim braquiária se mostrou eficiente apenas na fitorremediação de solos com contaminação correspondente ao grau de intervenção, sendo o Zn o metal com melhor remoção. Para contaminações com concentrações superiores houve redução significativa da massa seca e sintomas de intoxicação com a morte das plantas.

Ambas as espécies não foram eficientes para fitorremediação de solos contaminados com cádmio.

\section{Referências}

AGUNBIADE, F. O., OLU-OWOLABI, B. I; ADEBOWALE, K. O., Phytoremediation potential of Eichornia crassipes in metal-contaminated coastal water,. Bioresource Technology, v. 100, n. 19, p. 4521-4526, 2009.

CETESB - Companhia de Tecnologia de Saneamento Ambiental. Decisão de Diretoria n ${ }^{\circ} 195$ 2005, de 23 de Novembro de 2005. Dispõe sobre a aprovação dos valores orientadores para solos e águas subterrâneas no Estado de São Paulo, São Paulo, 2005.

CUNNINGHAM, S.D.; ANDERSON, T.A.; SCHWAB, P. e HSU, F.C.; Phytoremediation of soils contaminated with organic polluants. Advance in Agronomy, v.56, p. 55-114, 1996.

GAETKE, L.M., CHOW, C.K.. Copper toxicity, oxidative stress, and antioxidant nutrients. Toxicology, v. 189, p.147-163, 2003.

GARBISU, C.; ALKORTA, I. Phytoextraction: a cost effective plant-based technology for the removal of metals from the environment. Bioresource Technology, v. 77, p. 229 -236, 2001.

HONG-MING LIANG, TING-HSIANG LIN , JENG-MIN CHIOU, KUO-CHEN YEH, Model evaluation of the phytoextraction potential of heavy metal hyperaccumulators and non-hyperaccumulators Environmental Pollution, v.157, p.1945-1952, 2009. 
HUANG, J.W.; CUNNINGHAM, S.D. Lead phytoextraction: species variation in lead uptake and translocation. New Phytologist, v. 134, p 75-84, 1996.

KHAN, A.G.; KUEK, C.; CHAUDHRY, T.M.; KHOO, C.S; HAYES, W.J. Role of plants, mycorrhizae and phytochelators in heavy metal contaminated land remediation. Chemosphere, v.41, p.197207, 2000.

McGrath, S.P., Zhao, F.J., Lombi, E., 2002. Phytoremediation of metals, metalloids, and radionuclides. Adv. Agron. v. 75,p. 11 on

MAINE, M.A., SUN E, N.L., LAGGER, S.C., Chromium bioaccumulation: comparison of the capacity of two floating aquatic macrophytes. Water Research v. 38, p. 1494-1501, 2004

MALAVOLTA, E. VIITI, G. C., OLIVEIRA, S. A.. Avaliação do Estado Nutricional das Plantas - Princípios e Aplicações.,2 ${ }^{a}$ Ed., Piracicaba: Potafos, 1997, 319 p.

MULLIGAN, C.N., YONG, R.N., GIBBS, B.F. Remediation technologies for metal contaminated soils and groundwater: an evaluation, Eng. Geol. v. 60, p.193-207, 2001.

MULLIGAN, C.N.; YONG, R.N. \& GIBBS, B.F. Remediation technologies for metal-contaminated soils and groundwater: An evaluation. Engineering.Geology, v. 60, p. 193-207, 2001.

Pivetz, B.E., 2001. Phytoremediation of Contaminated Soil and Ground Water at Hazardous Sites. Ground Water Issue, EPA/540/S-01/500.

RASKIN, I.; KUMAR, PBA. N.; DUSHENKOV, S.; SALT, D.E. Bioconcentration of heavy metals by plants. Current opinions in Biotechnology, v.5, p.285-290, 1994

SHENGWEI PENG, QIXING ZHOU, ZHANG CAI, ZHINENG ZHANG, Phytoremediation of petroleum contaminated soils by Mirabilis Jalapa L. in a greenhouse plot experiment. Journal of Hazardous Materials v.168, p. 1490-1496, 2009.

SILVA, S.; SIQUEIRA, J.O. E SOARES, C.R.F.S. Fungos micorrízicos no crescimento e na extração de metais pesados pela braquiária em solo contaminado. Pesquisa Agropecuária Brasileira, v. 41, n. 12, p. 1749-1757, 2006.

SINGH, O.V.; Phytoremediation of toxic aromatic polluants from soil. Applied Microbiology and Biotechnology, v. 63, n. 2, p. 128-135, 2003.

SKINNER, K., WRIGHT, N., Porter-Goff, E., Mercury uptake and accumulation by four species of aquatic plants. Environmental Pollution, v. 145, p. 234-237, 2007.

UNITED STATES ENVIRONMENTAL PROTECTION AGENCY (US-EPA). Introduction to phytoremediation: EPA/600/R-99/107. Cincinatti: National Risk Management Research Laboratory, 2000

WEI, S., SILVA, J. A. T., ZHOU, Q., Agro-improving method of phytoextracting heavy metal contaminated soil, Journal of Hazardous Materials, v. 150, p. 662-668, 2008.

ZEITOUNI, C. F., BERTON, R.S.; ABREU, C. A.. Fitoextração de cádmio e zinco de um latossolo vermelho-amarelo contaminado com metais pesados. Bragantia, Campinas, v.66, n.4, p.649$657,2007$. 